\title{
Modified method of identification of mutual fractional-order inductance
}

\author{
Agnieszka Jakubowska-Ciszek ${ }^{1 *}$, Janusz Walczak ${ }^{1}$ \\ ${ }^{1}$ Faculty of Electrical Engineering, Silesian University of Technology, ul. Akademicka 10, 44-100 Gliwice
}

\begin{abstract}
The paper presents a method for identifying the parameters $M_{\gamma}, \gamma$ of a fractional-order transformer, which parameters $L_{\beta 1}, \beta_{1}, L_{\beta 2}, \beta_{2}$ have been previously determined. This method is based on the measurement of the phase resonance frequency in a few systems containing: the investigated fractionalorder transformer and two standard capacitors. The measurements need to be performed only for one series opposite-aiding connection of the fractional-order transformer. The dependencies allowing the determination of the fractional-order mutual inductance parameters have been given
\end{abstract}

\section{Introduction}

There are many works devoted to the analysis of systems with fractional-order elements $L_{\beta}, C_{\alpha}$, their realization and parameter identification, e.g. [1-3].

For several years, there has been a rapid growth of interest in fractional differential-integral calculus application in describing fractional-order magneticallycoupled coils systems [4-6]. The work [4] describes the concept and properties of such fractional-order coupled inductances. In [5], the electromagnetic Maxwell equations of the fractional-order mutual inductance are analyzed. The wireless power transmission system has been modeled as a fractional-order coupled coils system in [6]. The existence of fractional-order coupled coils (fractional-order transformer) implies the need to determine the parameters of the fractional-order elements. In [7], a method has been proposed for parameters identification of the fractional-order coils with an iron core, which is based on the approximation of the transient response to the unit-step voltage using the least squares method.

The paper is an extension and continuation of [8], where the new method for the identification of all the parameters $L_{\beta 1}, \beta_{1}, L_{\beta 2}, \beta_{2}, M_{\gamma}, \gamma$, of the fractional-order coupled inductances, has been proposed. The paper presents a proposal for a modified method of the identification of the fractional-order parameters $M_{\gamma}, \gamma$ of the mutual inductance, based on the phase resonance phenomenon in the series circuit of the class $R L_{\beta} C_{\alpha}$, compared to [8], without the need of the input impedance measurement in the combination of series and opposite-aiding connection of the transformer system.

\section{Modification proposal}

The equivalent circuit of the system for the parameters $\gamma$, $M_{\gamma}$ determination of the fractional-order mutual inductance, is shown in Fig. 1.

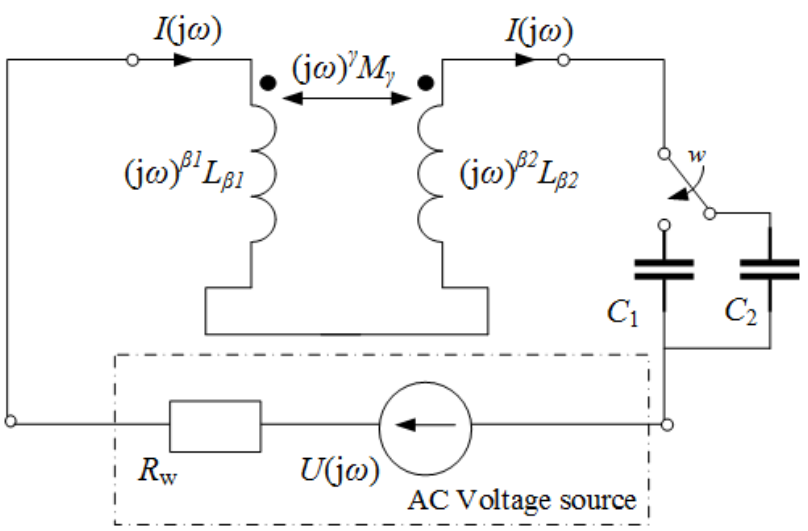

Fig. 1. The equivalent circuit for the fractional-order parameters identification by resonance method.

The circuit from Fig. 1 is supplied by the sinusoidal voltage source of adjustable frequency.

The circuit impedance, seen from the source terminals, is given by a formula:

$$
Z(\mathrm{j} \omega)=R+(\mathrm{j} \omega)^{\beta 1} L_{\beta 1}+(\mathrm{j} \omega)^{\beta 2} L_{\beta 2}-2(\mathrm{j} \omega)^{\gamma} M_{\gamma}-\mathrm{j} \frac{1}{\omega \mathrm{C}}
$$

where: $R$ - the equivalent resistance of the series connection of the coil resistances.

Transforming, the real and imaginary part of the impedance is: 


$$
\begin{aligned}
\operatorname{Re}\{Z(\mathrm{j} \omega)\}=R+\omega^{\beta 1} L_{\beta 1} \cos \left(\frac{\beta_{1} \pi}{2}\right) & +\omega^{\beta 2} L_{\beta 2} \cos \left(\frac{\beta_{2} \pi}{2}\right)- \\
& -2 \omega^{\gamma} M_{\gamma} \cos \left(\frac{\gamma \pi}{2}\right)
\end{aligned}
$$

and:

$$
\begin{array}{r}
\operatorname{Im}\{Z(\mathrm{j} \omega)\}=\omega^{\beta 1} L_{\beta 1} \sin \left(\frac{\beta_{1} \pi}{2}\right)+\omega^{\beta 2} L_{\beta 2} \sin \left(\frac{\beta_{2} \pi}{2}\right)- \\
-2 \omega^{\gamma} M_{\gamma} \sin \left(\frac{\gamma \pi}{2}\right)-\frac{1}{\omega C}
\end{array}
$$

Parameters $L_{\beta 1}, \quad \beta_{1}, \quad L_{\beta 2}, \quad \beta_{2}$ were determined previously, according to the procedure described in [8]. The circuit from Fig. 1 should be brought into phase resonance state, which will occur when the phase shift between the voltage measured on the series connection of the magnetically-coupled coil system as well as the capacitor $C_{1}$ and the current, will be equal to zero.

The measurement described above should be repeated twice, for two values of classic capacitances $C_{1}, C_{2}$ and the detection of the phase resonance frequency values, $\omega_{1}, \omega_{2}$ respectively.

Next, using the general phase resonance condition $\operatorname{Im}\{Z(\mathrm{j} \omega)\}=0$ and transforming, we get the relation:

$$
\left(\frac{\omega_{1}}{\omega_{2}}\right)^{\gamma}=\frac{\omega_{1}^{\beta 1} L_{\beta 1} \sin \left(\frac{\beta_{1} \pi}{2}\right)+\omega_{1}^{\beta 2} L_{\beta 2} \sin \left(\frac{\beta_{2} \pi}{2}\right)-\frac{1}{\omega_{1} C_{1}}}{\omega_{2}^{\beta 1} L_{\beta 1} \sin \left(\frac{\beta_{1} \pi}{2}\right)+\omega_{2}^{\beta 2} L_{\beta 2} \sin \left(\frac{\beta_{2} \pi}{2}\right)-\frac{1}{\omega_{2} C_{2}}}
$$

Then the value of the parameter $\gamma$ can be determined as:

$$
\gamma=\log _{\left(\frac{\omega_{1}}{\omega_{2}}\right)}\left(\frac{\omega_{1}^{\beta 1} L_{\beta 1} \sin \left(\frac{\beta_{1} \pi}{2}\right)+\omega_{1}^{\beta 2} L_{\beta 2} \sin \left(\frac{\beta_{2} \pi}{2}\right)-\frac{1}{\omega_{1} C_{1}}}{\omega_{2}^{\beta 1} L_{\beta 1} \sin \left(\frac{\beta_{1} \pi}{2}\right)+\omega_{2}^{\beta 2} L_{\beta 2} \sin \left(\frac{\beta_{2} \pi}{2}\right)-\frac{1}{\omega_{2} C_{2}}}\right) .
$$

However, the parameter $M_{\gamma}$ can be determined by substituting the obtained value of the coefficient $\gamma$ with the formula (3) for one of the performed measurements, for example for:

$$
M_{\gamma}=\frac{\omega_{1}^{\beta 1} L_{\beta 1} \sin \left(\frac{\beta_{1} \pi}{2}\right)+\omega_{1}^{\beta 2} L_{\beta 2} \sin \left(\frac{\beta_{2} \pi}{2}\right)-\frac{1}{\omega_{1} C_{1}}}{2 \omega_{1}^{\gamma} \sin \left(\frac{\gamma \pi}{2}\right)}
$$

The described algorithm has been illustrated with a simulation example.

\section{Example}

The circuit from Fig. 1 has been supplied from a source with an adjustable frequency value, for which the input voltage value has been assumed $U(\mathrm{j} \omega)=1 \mathrm{~V}$.

Parameters of the primary and secondary side of the transformer have been determined according to the procedure diagram [8] and were respectively: $\beta_{1}=0.503$, $L_{\beta 1}=8.813 \mathrm{mH} \cdot \mathrm{s}^{(1-\beta)}, \beta_{2}=0.502, L_{\beta 2}=3.113 \mathrm{mH} \cdot \mathrm{s}^{(1-\beta)}$. For two capacitors with known capacitances $C_{1}=10 \mathrm{mF}$, $C_{2}=3,53 \mathrm{mF}$ in the investigated circuit, as in Fig. 1, two values of resonance frequencies $f_{1}=100 \mathrm{~Hz}, f_{2}=200 \mathrm{~Hz}$ have been recorded. From the dependences (4) and (5), the searched values of the fractional-order parameters of the mutual inductance have been determined:

$$
\gamma=0.503
$$

and:

$$
M_{\gamma}=1.554 \mathrm{mH} \cdot \mathrm{s}^{(1-\gamma)}
$$

\section{Summary}

The paper proposes a modified method, compared to the method presented in [8], for identifying $M_{\gamma}, \gamma$ parameters of a fractional-order transformer. This method is based on the measurement of the phase resonance frequency in a circuit containing the analyzed transformer and two switchable standard capacitors. The dependencies allowing the determination of the fractional-order mutual inductance parameters have been given, on the basis of the described measurements.

The advantage of the modified method for determining the fractional-order parameters is the fact, that only one series opposite-aiding connection of the fractional-order coupled coils is enough to determine the searched parameters. The need to measure the input impedance of the circuit from Fig. 1 is also avoided.

\section{References}

1. A.S. Elwakil, IEEE Cir. Syst. Mag, 10, 40-50 (2010)

2. M. Sowa, Cir. Sys. \& Sign. Proc., 37, 4695-4727 (2018)

3. Ł. Majka, Bul. Pol. Acad. Sci., Tech. Sci., 66, $467-$ 474 (2018)

4. A. Soltan, A.G. Radwan, A.M. Soliman, Int. Jour. on Circ. Th. and Appl., 44, 85-97 (2015)

5. G. Liang, J. Hao, D. Shan, Jour. of Mod. Phys., 8, 2209-2218 (2017)

6. X. Shu, B. Zhang, Energies, 11(1774), 1-9 (2018)

7. P. Xia, S. Liang, 1st Int. Conf. on Inf. Tech., Inf. Sys. and El. Eng. (ICITISEE), 31-36 (Yogyakarta, Indonesia, 2016)

8. A. Jakubowska-Ciszek, J. Walczak, Pozn. Univ. Tech. Ac. Jour. El. Eng., 97, 169-180 (2019) 